\title{
El servicio de documentación de un medio impreso regional: evolución en el diario Levante-EMV
}

\author{
Por Marcos Ros-Martín y Alfonso Rodero-Susiac
}

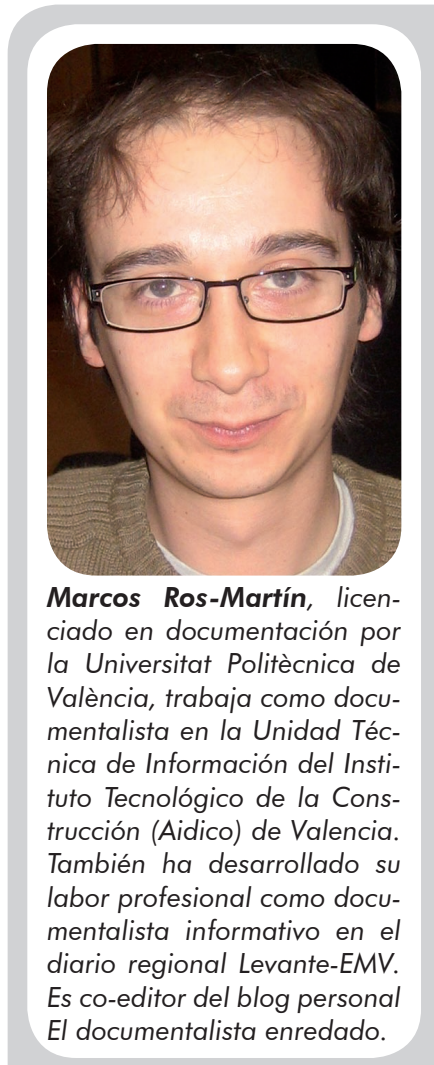

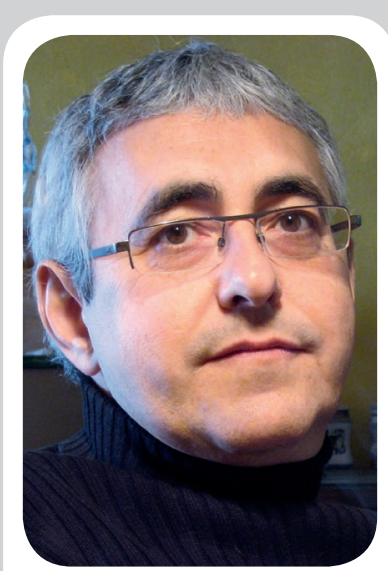

Alfonso Rodero-Susiac es diplomado en biblioteconomía y documentación por la Universidad de Murcia. Máster en periodismo y comunicación por la Universitat de València en colaboración con el diario Levante-EMV. Desde 1984 es el responsable del departamento de Documentación del diario Levante-EMV.

Resumen: Se estudia la evolución histórica de los procesos de gestión documental en el diario regional Levante-EMV desde su privatización en 1984 hasta nuestros días. Se señalan las distintas etapas que se observan tradicionalmente en los medios de comunicación impresos y se superponen a la evolución propia del diario para trazar una visión de conjunto. Asimismo, se detallan los modos de tratamiento documental utilizados dentro del diario dependiendo de los formatos de los documentos tanto en su gestión de forma manual como en la actual integración del archivo en el proceso productivo digital del diario.

Palabras clave: Medios de comunicación social, Diarios, Prensa regional, Servicios de documentación, Centros de documentación de prensa, Imagen digital, Gestión de la documentación fotográfica, Levante-EMV.

Title: A regional newspaper archive: the evolution of Levante-EMV

Abstract: Summary of the historical evolution of document management processes in the Spanish regional newspaper Levante-EMV from its privatization in 1984 to the present. First, it points out the historical stages that have been traditionally identified in print media archives, overlaid with the evolution of Levante-EMV. It then details the methods of document management that have been used in the newspaper archive, from manual document management to the integration of the archive in digital production process.

Keywords: Mass media, Newspapers, Regional newspaper, Press archives, News libraries, Document management, Digital image, Levante-EMV.

Ros-Martín, Marcos; Rodero-Susiac, Alfonso. "El servicio de documentación de un medio impreso regional: evolución en el diario Levante-EMV”. El profesional de la información, 2009, mayo-junio, v. 18, n. 3, pp. 316-322.

DOI: 10.3145/epi.2009.may.09

EN EL ENTORNO ANGLOSAJÓN los archivos de los medios de comunicación impresos han sido considerados una parte fundamental de cualquier periódico, a los que aportan un valor esencial.

Durante los 80 los nuevos periódicos que se lanzaban en el Reino Unido consideraban que el archivo constituía una "misión crítica" en su concepción, llegándose al extremo de que la primera persona a la que contrató el diario Today fue un documentalista. Por supuesto, esta visión también se da en las cabece- ras más antiguas donde el centro de documentación, o morgue ${ }^{1}$, disfruta de un valor icónico puesto que por ellos han pasado innumerables generaciones de periodistas utilizándolo como fuente histórica de informaciones.

En España las empresas editoras de medios de comunicación regionales no han dedicado demasiados recursos a la gestión de la documentación gráfica o textual que generan en su trabajo diario. De hecho, el fondo fotográfico suele ser una competencia que se deja a los reporteros gráficos, que deben gestionar los documentos de la mejor forma posible, mientras que la documentación impresa suele ser competencia, por su propio interés, del propio redactor que realiza su seguimiento o que se encuentra en una sección informativa determinada (Aquesolo-Vargas, 1996; Amat, 1995).

El trabajo dentro de una sección de Documentación de un medio de comunicación social impreso es diverso. Como bien recogen Gómez-Vázquez (1997) y Rico-Jerez (2002), los trabajos documentales que se realizan en un periódico dis- 


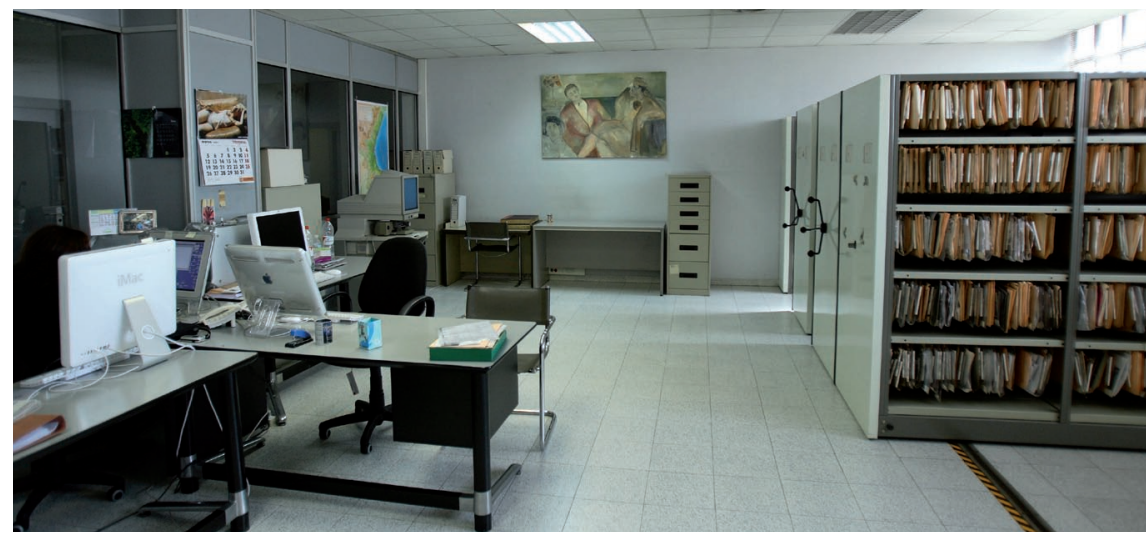

Instalaciones del archivo

tan mucho del trabajo que se puede realizar en una biblioteca o un archivo. Además de las tareas propias de descripción del fondo gráfico y de las informaciones y artículos que se publican en el periódico, o de la gestión de pequeñas bibliotecas de obras de referencia y manuales, los documentalistas se encargan de hacer búsquedas de información complejas y, según los casos, de la confección de perfiles e informaciones periodísticas.

Los documentalistas informativos, al igual que sucede con los medios de comunicación, se enfrentan a uno de sus mayores retos: la introducción de internet en todas las etapas del proceso productivo a la hora de elaborar una noticia y ante la que, desgraciadamente, el trabajo de los documentalistas puede contemplarse como algo innecesario para una empresa editora de un medio de comunicación.

\section{Períodos de la documentación en el diario Levante-EMV}

Desde la aparición de los medios de comunicación impresos a finales del siglo XIX, las distintas tareas y funciones que se han asignado a los documentalistas informativos han variado a lo largo del tiempo, así como la adecuación de las nuevas tecnologías a las tareas de recuperación de información. Sin querer ser exhaustivos, la mayoría de los autores (González-Quesada, 1995; Hernández-Pérez, 2000; Delgado-
Soler, 1999) identifican los mismos períodos: 1954)

- Carpetas del cortar y pegar (1954-1972)

- Microfilmación y bases de datos referenciales (1972-1982)

- Cd-rom y bases de datos a texto completo (1982-1992)

- Texto completo e interconexión de sistemas (1993-1998)

Finalmente, teniendo presentes las preferencias actuales en el
- Índices temáticos (1860- trabajo que se realiza dentro de las redacciones de los medios de comunicación, habría que añadir una etapa más:

- La integración del archivo en el proceso productivo digital del diario (1998-) ${ }^{2}$.

En el caso del diario Levante$E M V(E M V=E l$ mercantil valenciano) estas etapas se adoptaron en períodos de tiempo mucho más cortos dependiendo de los recursos y la tecnología disponible en cada momento. Sin embargo, nos parece interesante tratar de arropar las distintas etapas vividas en la sección de Documentación siguiendo este esquema.

\section{a. Carpetas colgantes (1984-1996)}

En el diario Levante-EMV no había una persona dedicada a tiempo completo a la organización del archivo hasta que se optó por ello coincidiendo con la privatización de la empresa ${ }^{3}$ en 1984. Durante el período anterior, el archivo lo formaban varios archivadores en los que se almacenaban fotografías sin

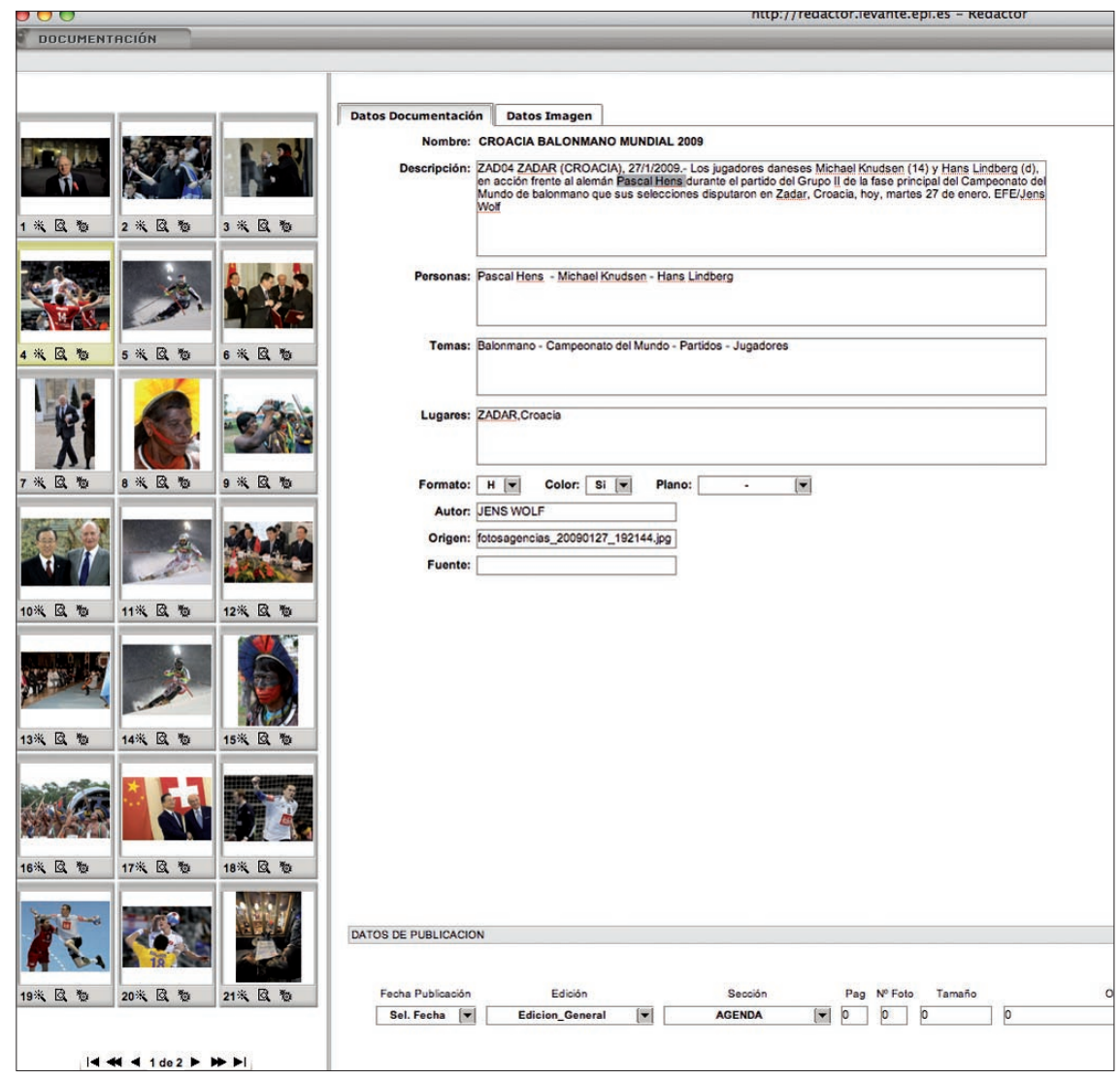

Interfaz de Redactor para el servicio de Documentación 


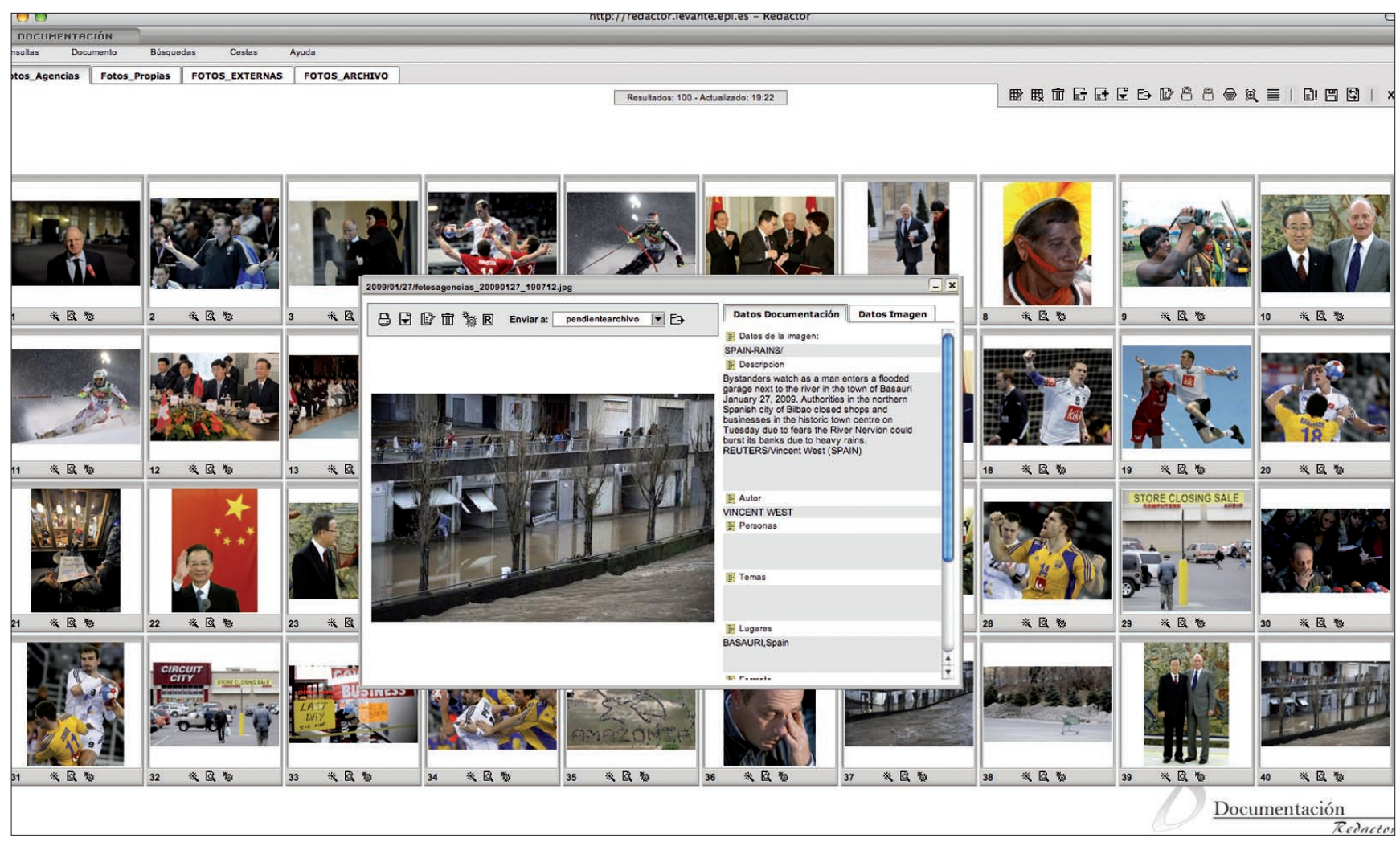

Interfaz de Redactor

ningún orden preestablecido a los que, dependiendo del momento y las necesidades informativas, los redactores acudían a revisar y seleccionar la que considerasen adecuada. Hay que señalar que se trata de una época en la que apenas se tiene respeto por los documentos gráficos y, en ocasiones, lo que hoy se denomina siluetear una fotografía entonces suponía recortar la copia con unas tijeras sin tener presente que, casi siempre, ese documento era único ${ }^{4}$.

Tras la incorporación de un documentalista a la redacción, la situación de la documentación gráfica comienza a mejorar de forma significativa. Por un lado, se acomete un trabajo de repaso, expurgo y reclasificación de todo el material fotográfico anterior, a la vez que se trabaja en la sistemática para la descripción y clasificación del nuevo material que se va produciendo en el día a día. En este período nos encontramos fundamentalmente con dos tipos de material fotográfico: fotografías en blanco y negro y telefotografías.
Para la organización de todo el material gráfico, y aprovechando el cambio de sede en 1988, el diario Levante-EMV adopta el sistema de carpetas colgantes utilizando como mobiliario de archivo los compactos (grandes estanterías con ruedas), comenzando a utilizar un índice temático para localizar los documentos. Este índice temático de elaboración propia agrupa en 150 páginas una clasificación estructurada en 9 apartados (cuatro de ellos alfabéticos) mediante una serie de divisiones y subdivisiones junto a reenvíos a distintos epígrafes. Cada epígrafe lleva aparejado un código topográfico para la localización de los originales en los compactos.

El análisis de las fotografías varía dependiendo del tipo y del contenido de las mismas, aunque por entonces se considera que es indispensable que figuren los siguientes datos: fecha de realización, autor de la fotografía, fecha de publicación, localización fotográfica, personas y objetivos que muestra y, finalmente, el contenido subjetivo o connotación que refleja una percepción personal.
La previsión de aumento del fondo documental se calculaba sobre una base de 200 fotografías diarias de los reporteros gráficos de la casa, mientras se almacenaba también buena parte de las fotografías de agencia que entonces se recibían mediante el sistema de telefotografía. En la actualidad, el fondo documental fotográfico en formato físico, dando una cifra aproximada, puede ascender a unos 800.000 documentos.

La incorporación de un nuevo miembro a la sección de Documentación a finales de los 80 permitió, entre otras cosas, poder ampliar el servicio hacia el material impreso que se publicaba en el diario. De esta manera, las informaciones se recortaban y fotocopiaban según sus temas y, de la misma forma que se hacía con las fotografías, se almacenaban en carpetas o en sobres dependiendo del volumen de informaciones publicadas. Esta nueva actividad supuso un avance para los redactores a la hora de abordar los nuevos trabajos periodísticos y hoy en día, tras la implantación de la 


\section{"Se ha pasado del almacenamiento sin orden, a la estructuración y descripción de los fondos documentales digitalizados"}

documentación digital, es una tarea ampliamente aceptada y bien considerada dentro de la redacción.

La clasificación de carpetas y sobres se fundamentó sobre Personas, Organismos (empresas, partidos políticos, administraciones, organismos públicos e instituciones), Temas específicos (educación, economía, política, temas locales, sanidad, cultura, sucesos, etc.), y Localizaciones geográficas.

b. Microfilmación y bases de datos referenciales (1991-2005)

La compra de una nueva rotativa en 1987 que permitía la publicación de periódicos con fotografías en color supuso el comienzo de la utilización de las diapositivas tanto en paso universal $(24 \times 36 \mathrm{~mm})$ como en paso grande $(60 \times 70 \mathrm{~mm})$. El inicio de la publicación del magazine La Cartelera, en 1990, fomentó un importante crecimiento de este formato fotográfico.

A pesar de que los criterios de análisis y selección eran los mismos que en el caso de fotografías impresas, no sucedió así con su tratamiento y descripción, ya que comenzaron a utilizarse en un primer momento fichas de cartulina, que heredaron los campos de descripción anteriormente citados, para posteriormente utilizarse una base de datos informática gestionada con el programa Filemaker para su más rápida y eficaz localización y recuperación. Por otro lado, su almacenamiento se realiza de forma similar al de las fotografías, en fundas colgantes específicas para diapositivas, distribuidas en compactos

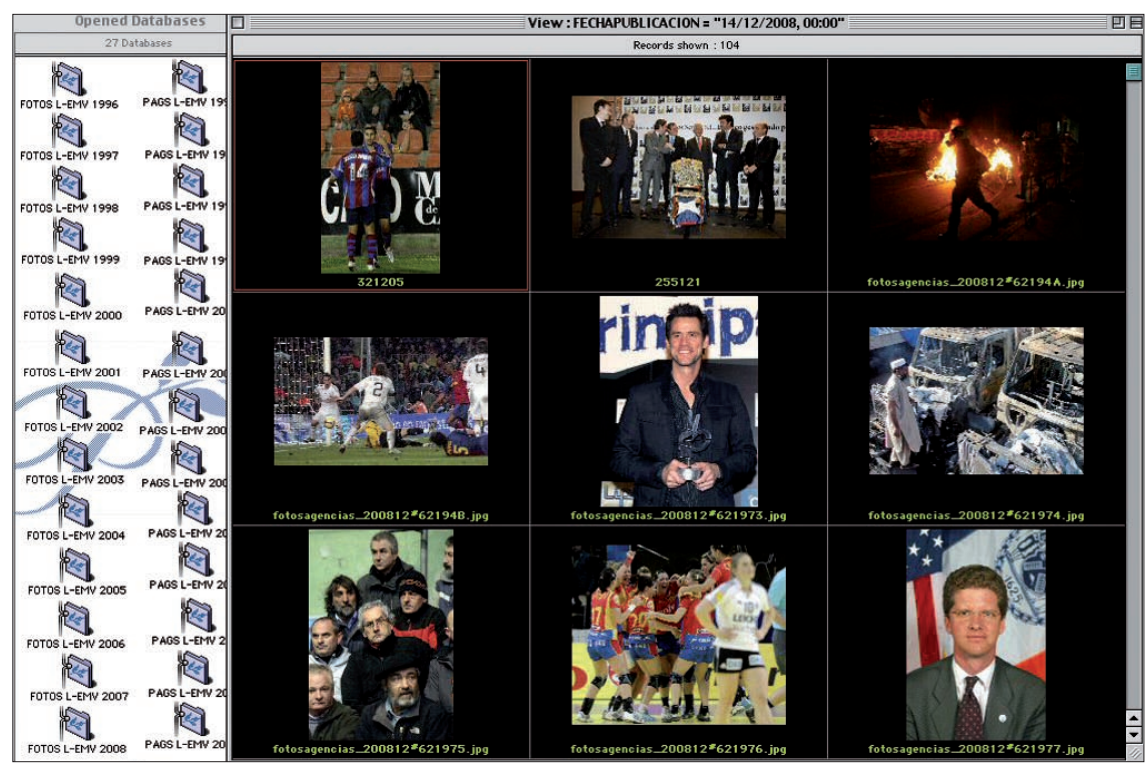

Página de resultados de Phrásea

aunque en este caso ya no es necesario agruparlas temáticamente.

Durante este período y teniendo presente la larga historia del diario, se llegó a un acuerdo de colaboración con la Biblioteca Municipal de Valencia que supuso tener microfilmados todos los ejemplares del diario publicados desde su fundación. En la actualidad, además de disponer de los ejemplares en formato microfilm, se encuentran digitalizados en pdf y pueden ser consultados mediante una base de datos sencilla referencial desde una intranet.

\section{"La documentación de las fotografías se realiza mediante un vocabulario controlado"}

\section{c. Bases de datos documentales (1996-)}

En junio de 1996 la sección de Documentación de Levante-EMV inicia una nueva etapa en cuanto al tratamiento del fondo documental, puesto que, además de alcanzar su plantilla la cantidad de cuatro miembros, se inicia la informatización de los procesos que realizan. Los ordenadores comenzaron a inundar la redacción a principios de los 90 para la composición y edición de textos, sin embargo durante ese período el producto informativo y las fotografías llegaban al archivo de forma analógica a pesar de los tímidos esfuerzos de gestionar los fondos con una base de datos referencial.

Tras la compra del gestor documental Phrásea ${ }^{5}$, la gestión de fotografías y de la documentación se informatiza completamente. Hay que recalcar que el gestor documental era un producto caro y la limitación en la compra de licencias fue un impedimento para que los redactores pudiesen realizar sus propias consultas, que debían pasar por el filtro del documentalista. Existía la posibilidad de utilizar terminales con copias de software cliente para consultar las bases de datos existentes, cosa que resultaba muy útil para las secciones de Diseño, Cierre o las distintas delegaciones comarcales, pero por las razones antedichas no se extendió su uso a toda la Redacción.

Para la indización de las fotos se tomaban los metadatos de los campos IPTC $C^{6}$ cumplimentados por los fotógrafos del diario o de las agencias, mientras que para la documentación impresa se extraía el texto completo de los ficheros en pdf. Los campos de descripción se redujeron 


\section{"Además del vocabulario controlado se requiere en ocasiones la utilización de una frase descriptiva del contenido"}

a los estrictamente imprescindibles, de tal manera que en el caso de las fotografías disponemos de Personas, Temas, Lugares, Descripción, Modo (horizontal o vertical, blanco y negro o color), FechaPublicación, Página (de publicación), OtrasPublicaciones, Autor, FechaCreación, FechaEntrada, Documento (Id) y en el caso de los documentos textuales disponemos de Personas, Temas, Lugares, FechaPublicación, Página, Texto y Género (reportaje, opinión, editorial, noticia).

Para la documentación de las fotografías se usa un vocabulario de palabras clave, aunque que con frecuencia también se hace necesaria la utilización de una frase descriptiva del contenido, sobre todo con los documentos textuales. La necesidad de ir más allá en la descripción del contenido mediante un vocabulario controlado se convierte en acuciante con la propia dinámica de algunas informaciones, particularmente las políticas y las de sucesos y tribunales, por la cantidad de réplicas y contrarréplicas que se producen, así como por el seguimiento que ha de realizarse a los hechos noticiosos.

En otro orden de cosas hay que señalar que a lo largo de los años las distintas versiones del software y sus mejoras han permitido una mayor agilidad a la hora de gestionar los documentos. Así, en un principio, los fondos fotográficos y documentales se encontraban en servidores separados, cada uno utilizando un jukebox de 100 cd-roms que contenían los documentos (1996-2002), hasta el actual servidor que es capaz de gestionar las 25 bases de datos utilizadas. Para el

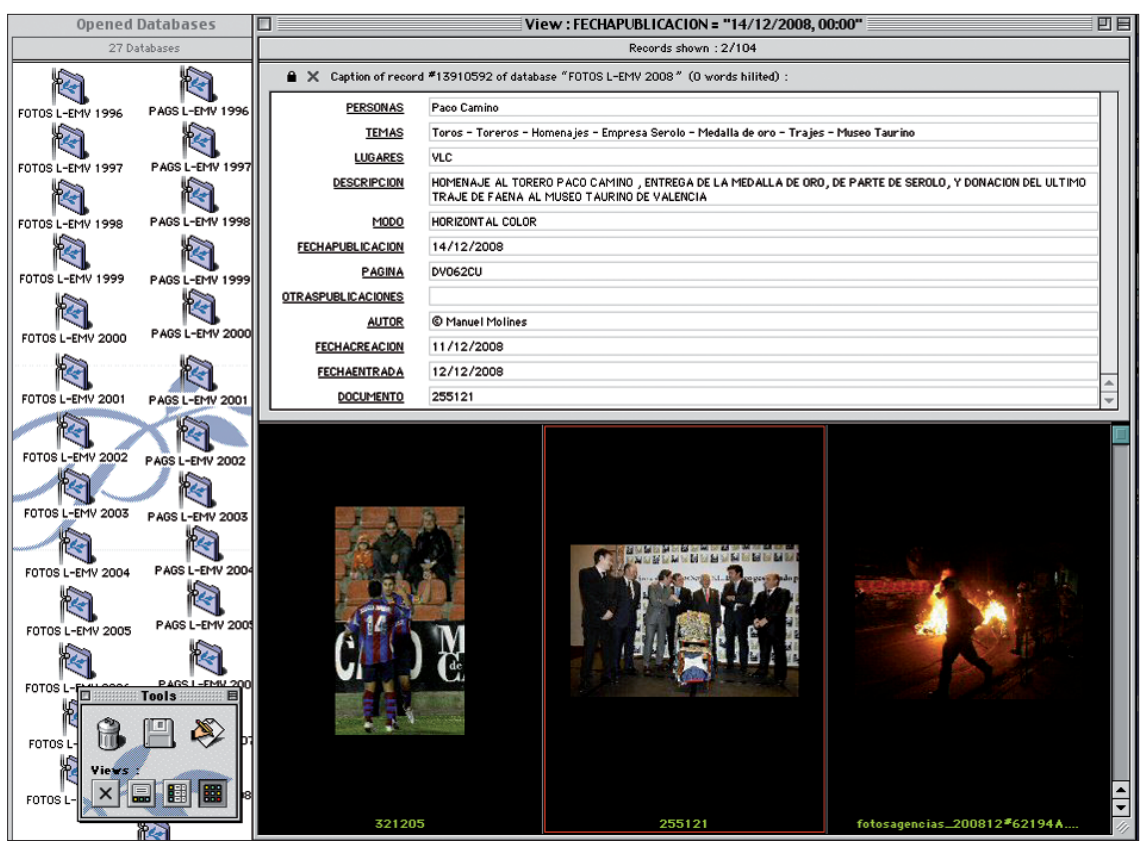

Página de resultados de Phrásea

almacenamiento de los documentos se utilizó primero un $R A I D^{7}$ y posteriormente discos duros extraíbles.

\section{"Las mejoras en el software han permitido mayor agilidad para gestionar los documentos"}

La posibilidad de crear distintas bases de datos permitió la división de los documentos por año de trabajo, de manera que cada año disponía de su base de datos correspondiente dividida en fotografías o documentos, consultables separada o conjuntamente.

\section{d. Integración del archivo en el proceso productivo digital del} diario (2009-)

Coincidiendo con la desaparición de la empresa Recursos en la Red (RENR), que aportaba soporte técnico al gestor documental del diario, Editorial Prensa Ibérica propietaria de Levante-EMV, adopta la decisión de crear una plataforma para la gestión de los flujos de trabajo dentro de los diarios del grupo. Esta plataforma se va llevando a cabo de forma paulatina implementando nuevas áreas de trabajo, añadiéndose nuevas funciones según se avanza en el proyecto. Así, si en un principio la solución desarrollada por RENR simplemente actuaba de contenedor de los teletipos y las fotografías recibidas por agencias, hoy las funciones se encuentran ampliadas hacia las tareas de archivo fotográfico, infográfico, alzado o maquetación de páginas, entre otras.

Esto supone un paso hacia la integración del servicio de Documentación dentro del proceso productivo del diario, lo que se está convirtiendo en toda una tendencia dentro de las empresas de comunicación social. De esta forma, finalmente, los redactores disponen de acceso a todo el fondo documental gráfico directamente, pudiendo ser ellos, más que el documentalista, quienes filtren los documentos que se van a publicar.

En el diseño de la aplicación, de nombre Redactor, se han tenido en cuenta los años de experiencia acumulados en el servicio de documentación y se ha elegido utilizar los mismos campos descriptivos. Esto también ha facilitado en gran medida el volcado de los documentos 


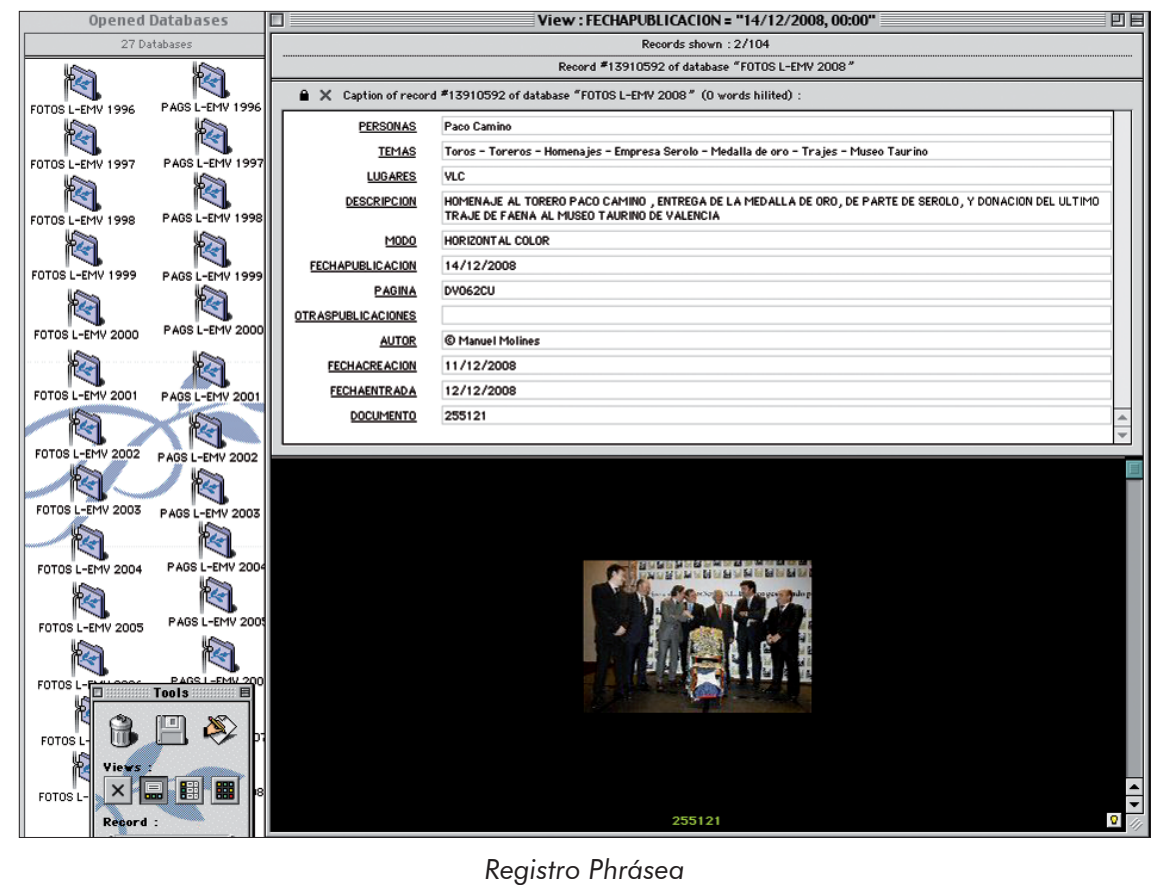

(casi 1 millón en el caso de fotografías digitales) que se gestionaban en el servidor de Phrásea, sin pérdidas de la información asociada a ellos. Hay que indicar, no obstante, que en el caso de la gestión de los documentos textuales la solución todavía es deficiente y se mantiene la utilización de Phrásea.

\section{Conclusiones}

La evolución en el tratamiento de la documentación dentro del Levante-EMV se ajusta a las etapas identificadas en el entorno anglosajón, diferenciándose de forma clara en los períodos temporales, siendo en nuestro caso mucho más cortos, pero que en etapas futuras se producirán de forma mucho más acompasada al resto del sector por razones competitivas a la hora de adoptar nuevas tecnologías.

De hecho, en tan sólo 25 años se ha pasado de una gestión del fondo documental gráfico completamente manual a la situación en la que todo el proceso de archivo es digital y está integrado plenamente dentro del proceso productivo del periódico. Capítulo aparte merece el tratamiento del fondo textual, bastante más complejo, que necesita de sólidas y potentes plataformas
Por otra parte, el esfuerzo por la sistematización en la descripción de los contenidos editados por el diario Levante-EMV ha supuesto un cambio en la cultura de la documentación dentro de la empresa, siendo percibida y valorada por los usuarios directos, esto es, los periodistas, tanto más por aquellos que hacen del rigor, la precisión y la contextualidad su norma. Por supuesto que todavía queda camino por recorrer, porque el trabajo del área de Documentación no es, en ocasiones, percibido como un beneficio tangible, olvidándose quizá del valor añadido que ofrece a la información, a la que aporta calidad y diferenciación respecto a su competencia, o del ingente ahorro de tiempo que supone a sus usuarios el tener al instante información precisa y relevante no localizable por otros medios.

\section{Notas} fondos documentales digitalizados, en los que la síntesis de lo importante, la criba de lo relevante y la extracción de lo pertinente es fundamental en el apoyo de la confección de un producto informativo de calidad, que huya del vuelo alicorto de tantos medios de comunicación que han convertido el corta y pega en identificador de su producto.
1. La utilización del término morgue para referirse al servicio de archivo en los medios de comunicación anglosajones deriva de los primeros usos que se dieron a estos centros, fundamentalmente la confección de perfiles y la búsqueda de material gráfico ante los decesos de personajes populares de la sociedad.

2. Sin querer ser exhaustivos, puesto que no es el objetivo de este artículo, nos referimos al hecho de que los nuevos workflows dentro de los

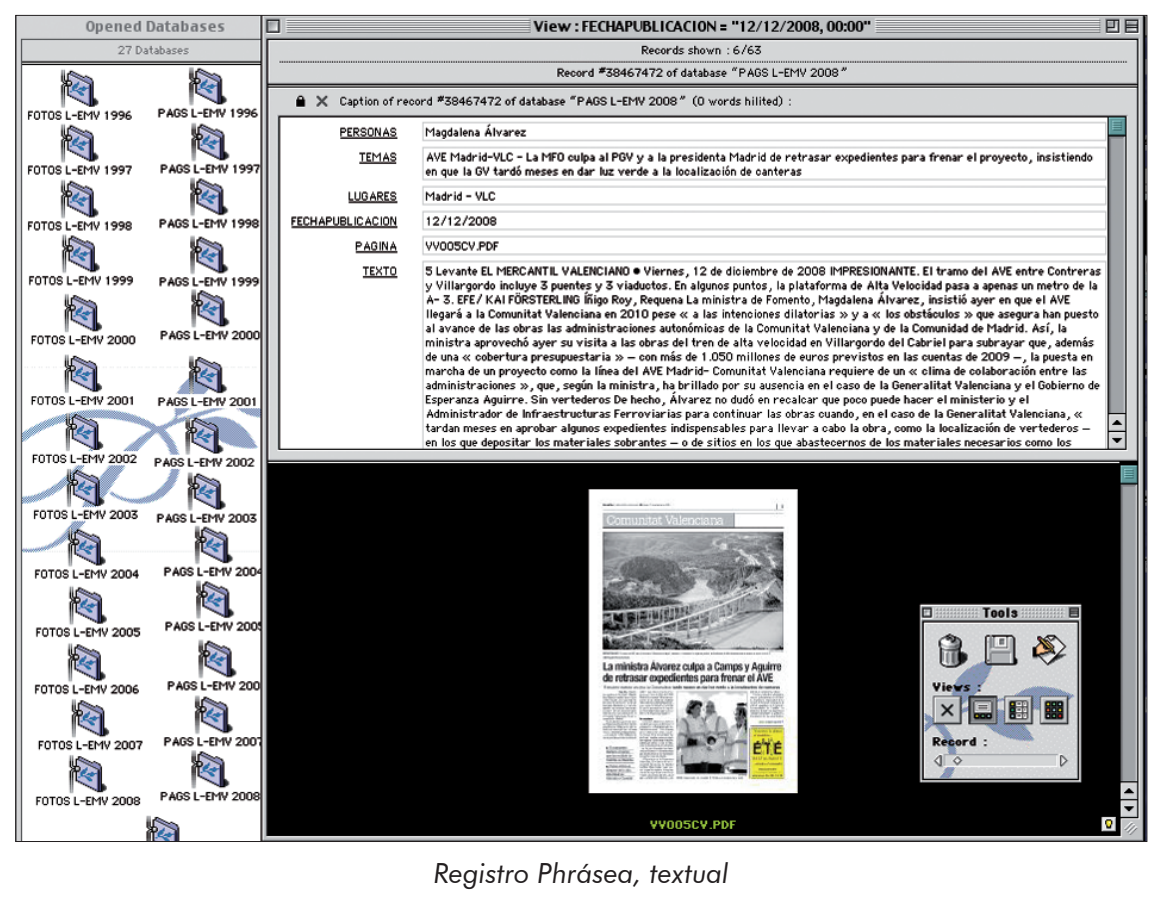

El profesional de la información, v. 18, n. 3, mayo-junio 2009 


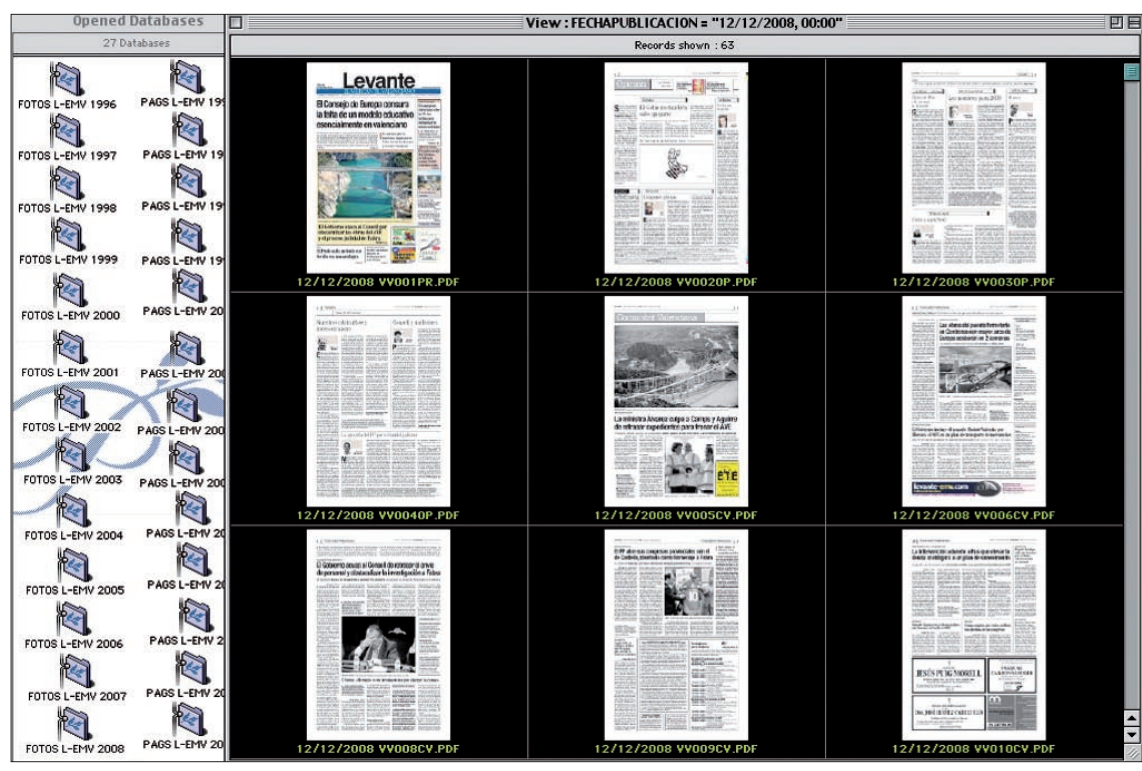

Resultados Phrásea, textual

medios de comunicación con la utilización de las tecnologías xml (NewsML) dentro de redacciones digitalizadas, permite la integración del archivo y del centro de documentación como un todo dentro de las tareas de producción del periódico impreso (Pascual, 2002).

3. Para más información sobre la historia del Levante-EMV recomendamos la monografía: Laguna, Antoni; Martínez, Francesc. Historia de Levante-El mercantil valenciano. Valencia Editorial Prensa Valenciana, 1992

4. En la documentación fotográfica analógica se consideraba como documento el negativo de la fotografía, mientras que la copia correspondía al positivo. En este caso, los negativos los almacenaba el fotógrafo mientras que el periódico se quedaba las copias puesto que la propiedad intelectual siempre es del fotógrafo aunque ceda parte de sus derechos al medio para su publicación.

5. El gestor documental Phrásea es propiedad de la empresa Alchemy. Actualmente se distribuye como plataforma web. Las versiones que hemos utilizado en el área de documentación son software a ejecutar en local en sus versiones de administrador, servidor o cliente.

http://www.alchemy.fr/

6. Los campos IPTC, definidos por el International Press Telecommunications Council, permiten la inserción de datos como el título del archivo, el dueño de los derechos de autor, la descripción del archivo y las palabras clave dentro del documento fotográfico digital.

7. RAID (Redundant Array of Independent Disks, "conjunto redundante de discos independientes") hace referencia a un sistema de almacenamiento que usa múltiples discos duros entre los que distribuye o replica los datos.

http://es.wikipedia.org/wiki/RAID

\section{Bibliografía}

Amat, Carlos B. "Algunas características de la documentación de prensa en la Comunidad Valenciana”. Métodos de información, n. 6-7, julioseptiembre 1995.

http://www.uv.es/biblios/mei6/cv9.html

Aquesolo-Vargas, José. "Situación de los servicios de Documentación en la prensa diaria de Andalucía". Cuadernos de documentación multimedia, n. 5, 1995.

http://www.ucm.es/info/multidoc/multidoc/revista/cuadern5/aquesolo.htm

Arundale, J.; Whitey, R. "Los sofisticados archivos permiten a los usuarios buscar mejor contenido". IFRA-Técnicas de prensa, febrero 2002, n. 2, pp. 14-18.

Delgado-Soler, Cinta. "Documentación en prensa”. En: García-Gutiérrez, Antonio (ed.), Introducción a la documentación informativa y periodística. Madrid: MAD, 1999, pp. 455-464.

González-Quesada, Alfons. "La evolución histórica de la documentación periodística". En: Fuentes Pujol, Maria Eulàlia (ed.), Manual de documentación periodística. Madrid: Síntesis, 1995 , p. 23.

Gómez-Vázquez, Miguel. "Servicio de archivo y documentación de un periódico: $E l$ mundo". Cuadernos de documentación multimedia, n. 67, 1997-1998.

http://www.ucm.es/info/multidoc/multidoc/revista/cuad6-7/gomez.htm

Hernández-Pérez, Antonio; Caridad-Sebastián, Mercedes. "Documentación escrita en los centros de documentación de los medios de comunicación". En: Moreiro González, José Anto- nio, Manual de documentación informativa. Madrid: Ediciones Cátedra, 2000, pp. 59-66.

López-García, Guillermo. "Ciberperiodismo en el ámbito local: el caso del diario LevanteEMV'. En: II Colóquio Brasil-Espanha sobre Cibermeios, São Paulo (Brasil), 17-18 noviembre 2008 .

http://www.cibermediosvalencianos.es/documentos/Levante-emv_GuillermoLopez_SaoPaulo2008.pdf

Pascual, Mari. "Heraldo de Aragón, pionero en el uso de Infópolis". IFRA-Técnicas de prensa, marzo 2002, n. 3, pp. 32-33.

Razquin, Pedro. "Situación de los centros de documentación en los medios de comunicación de Madrid". Cuadernos de documentación multimedia, n. 2, 1992.

http://www.ucm.es/info/multidoc/multidoc/revista/num2/prazquin.html

Rico-Jerez, Marta. "Los servicios de documentación del $A B C$ y La verdad de Murcia". En: Galdón, Gabriel. Teoría y práctica de la documentación informativa. Barcelona: Ariel, 2002, pp. 307-317.

Rodero-Susiac, J. Alfonso. "Organización de archivo fotográfico del diario Levante-EMV". En: II Seminari La documentació als mitjans d'informació: l'experiència multimèdia. Valencia, 7-9 marzo 1994, pp. 122-127.

Rodero-Susiac, J. Alfonso. "La documentación gráfica y textual en Levante de Castelló". Diario Levante de Castelló, Castellón, 5 julio 2001, pp. 48-49.

Ros-Martín, Marcos. "Breve historia de la gestión de la documentación en los medios de comunicación". El documentalista enredado, 1 agosto 2004.

http://www.documentalistaenredado.net/1/historia-gestion-documentacion-medios-comunicacion/

Shipside, Steve. "Los nuevos sistemas redefinen a los modernos documentalistas". IFRA-Técnicas de prensa, diciembre 2000, n. 12, pp. 22-23.

\section{Marcos Ros-Martín}

Instituto Tecnológico de la Construcción (Aidico), Paterna (Valencia)

marcos.ros@aidico.es

marcos@documentalistaenredado. net

\section{Alfonso Rodero-Susiac}

Departamento de Documentación, Diario Levante-EMV. alfonso@epi.es 